\title{
Multi-spectral imaging: a tool for the study of the area burned in wildfires.
}

\author{
Hernán Domingo Ramos, Artur Carnicer \\ Universitat de Barcelona (UB), Facultat de Física, Martí i Franquès 1, 08028 \\ Barcelona, Spain \\ E-mail: hdominra11@alumnes.ub.edu, artur.carnicer@ub.edu
}

18 August 2021

\begin{abstract}
Multi-spectral images provide powerful information to assess the effects of wildfires. Data can be processed, combined and analyzed in such a way that can contribute to suggest a protocol of action to help recover devastated areas. The objective of this paper is to describe methods to evaluate the surface burned in a forest fire. Combining the information recorded by the visible and infrared sensors of the Landsat 8 satellite, we can estimate the temperature of the surface after the wildfire. Finally, by comparing images recorded before and after the fire, we can estimate the surface burned.
\end{abstract}

Keywords: Multi-spectral imaging, digital image processing, image analysis, black-body radiation.

Submitted to: Eur. J. Phys.

\section{Introduction}

Climate change is one of the most serious threats that is facing the planet. Nowadays, large-scale forest fires are becoming more frequent, especially in dry areas with vegetation that can be found in Eastern Australia, California, or the Mediterranean countries, among others [1 3 . Moreover, even the Amazon rainforest can burn because of forest degradation and deforestation [4].

At the beginning of the summer of 2019, Catalonia suffered a severe heat wave. High temperatures, strong winds and the absence of rain led to the appearance of forest fires. On June 26th, a forest fire was declared in the Ribera de l'Ebre county, located $100 \mathrm{~km}$ west of Tarragona, burning an area of approximately $50 \mathrm{~km}^{2}[5]$. The fire was declared extinguished on July 7 th.

Landsat 8, a satellite operated by the US Geological Survey and NASA, can record multi-spectral images in 11 bands ranging from the visible to the long-wave infrared [6]. 
Nowadays, multi-spectral information plays a key role in the analysis of the effects of fire in forests. The objective of this paper is to describe a method to evaluate the surface burned in a forest fire. Starting from satellite images, we estimate the at-sensor temperature using a black body approach. Then, we assess the severity of the burned land using analysis methods on images recorded before and after the fire. Finally, we identify the damaged area using mathematical morphology operations. The values of the estimated area are compared with those reported by official sources.

Multi-spectral imaging is routinely used in the analysis of wildfires (see, for instance, [7] and references therein). Also, this topic is analyzed in textbooks on remote sensing [8] and several practical tutorials 9,10$]$. Other authors have considered topics that partially overlap with our work. Interestingly, Caridades and collaborators described educational experiences on basic image processing techniques and its application to the estimation of the area of a surface [11,12]. Moreover, multiple experiments involving infrared cameras have been described; we refer to $13-15$ to cite a few.

The paper is organized as follows: In section 2, we describe the image set required to perform the calculations. In section 3 we describe the procedure to perform geometrical changes on the images in such a way that no zero values appear. In Section 4, we estimate the at-sensor temperature using a black-body approach- Then, the differential normalized burn ratio is introduced in section 5. This index is useful to classify the severity of the damaged area. Finally, the burned area is calculated in section 6 . We present our conclusions in section 7 .

\section{Motivation}

Digital image processing is a basic technique [16] that is widely used in experimental physics. At the Universitat de Barcelona, Image Processing and Computer Vision (IPCV) is a 3 ECTS credit subject intended for junior or senior students in Physict $¥$. At this point, students have taken two courses (6 ECTS each) in coding techniques and computational physics and are skilled in Python. Depending on their academical profile (fundamental or experimental physics) they have some extra experience with Fortran or Labview.

IPCV is a problem-based introductory course focused on algorithm implementation. Around 30 highly motivated students take these credits every year. The students attend to lectures, computer labs and develop a computational project of their choice. They perform a bibliographical search, select a proper topic, develop the code and write a journal-like report. The present paper is an extended version of an original student project.

$\ddagger$ Note that students require 240 ECTS to graduate in a Spanish university. 


\section{Landsat 8 image set}

Satellite images are extremely useful for developing analysis tools that entitle us to understand the expansion of fires. Landsat 8 is an Earth observation satellite, launched on 11th February 2013. Landsat 8 comprises two sensors: The Thermal InfraRed Sensor (TIRS) and the multi-spectral Operational Land Imager (OLI) with 11 different channels available.

Landsat 8 datasets can be downloaded from the USGS Earth Explorer platform [17]. They comprise 11 geo-referenced images (corresponding to the bands described in Table 1), and two plain-text files of metadata. In this work we use images from bands 4, 5, 7 and 10, taken on August 14, 2019 and June 24, 2018.

Table 1: Landsat 8 spectral bands.

\begin{tabular}{lcc}
\hline \multicolumn{3}{c}{ OLI } \\
\hline & Spectral Band (Wavelength) & Spatial Resolution \\
\hline 1 - Coastal- Aerosol & $0.433-0.453 \mu \mathrm{m}$ & $30 \mathrm{~m}$ \\
- Blue & $0.450-0.515 \mu \mathrm{m}$ & $30 \mathrm{~m}$ \\
3 - Green & $0.525-0.600 \mu \mathrm{m}$ & $30 \mathrm{~m}$ \\
4 - Red & $0.630-0.680 \mu \mathrm{m}$ & $30 \mathrm{~m}$ \\
5 - Near Infrared (NIR) & $0.845-0.885 \mu \mathrm{m}$ & $30 \mathrm{~m}$ \\
- Short Wavelength Infrared (SWIR1) & $1.560-1.660 \mu \mathrm{m}$ & $30 \mathrm{~m}$ \\
7 - Short Wavelength Infrared (SWIR2) & $2.100-2.300 \mu \mathrm{m}$ & $30 \mathrm{~m}$ \\
8 - Panchromatic & $0.500-0.680 \mu \mathrm{m}$ & $15 \mathrm{~m}$ \\
9 - Cirrus & $1.360-1.390 \mu \mathrm{m}$ & $30 \mathrm{~m}$ \\
\hline & TIRS & $100 \mathrm{~m}$ \\
\hline 10 - Long Wavelength Infrared (LWIR1) & $10.30-11.30 \mu \mathrm{m}$ & $100 \mathrm{~m}$ \\
11 - Long Wavelength Infrared (LWIR2) & $11.50-12.50 \mu \mathrm{m}$ & \\
\hline
\end{tabular}

\section{Image preprocessing}

The size of Landsat 8 images is $7861 \times 7981$ pixels with a 16-bit pixel depth; each pixel describes an area of $30 \times 30 \mathrm{~m}^{2}$. They are delivered as $125 \mathrm{MB}$ TIFF files. This large amount of information cannot be easily handled and for this reason, we decided to reduce the number of pixels by a factor of 4 (i.e. $3930 \mathrm{x}$ 3990). Figure 1 shows Landsat images recorded on August 14th, 2019.

Moreover, the original images are geometrically manipulated to remove pixels with values equal to zero, that can distort the data range during the processing stage. However, this procedure introduces some resampling artifacts: in particular, the images display a slightly but noticeable shift. This effect can be compensated, as explained in section 5. The use of this approach is interesting from a pedagogical point of 
view, because important image processing techniques such as affine transform and 2Dcorrelation can be introduced. However, it is not required to modify the images since the zero values can be masked.

Homography matrix $\mathbf{H}$ relates the position of the pixels of the image before and after an arbitrary geometrical transformation.

$$
\left(\begin{array}{c}
x^{\prime} \\
y^{\prime} \\
1
\end{array}\right)=\mathbf{H}\left(\begin{array}{l}
x \\
y \\
1
\end{array}\right)=\left(\begin{array}{lll}
h_{11} & h_{12} & h_{13} \\
h_{21} & h_{22} & h_{23} \\
h_{31} & h_{32} & h_{33}
\end{array}\right)\left(\begin{array}{l}
x \\
y \\
1
\end{array}\right),
$$

where $(x, y, 1)$ and $\left(x^{\prime}, y^{\prime}, 1\right)$ refer to the pixel coordinates before and after the transformation [16]. Note that the third component (with value equal to 1 ) is used for mathematical convenience. Usually, matrix $\mathbf{H}$ is normalized in such a way that $h_{33}=1$.

In what follows, the size of the image is $N$ (rows) x $M$ (columns); the coordinates of the top-left and the bottom-right corners are $(0,0)$ and $(N-1, M-1)$ respectively (see Fig. 2). Then, the coordinates $(r, c)$ of the four vertices $\mathbf{v}_{\mathbf{1}}, \mathbf{v}_{\mathbf{2}}, \mathbf{v}_{\mathbf{3}}$ and $\mathbf{v}_{\mathbf{4}}$, are:

- $\mathbf{v}_{\mathbf{1}}$ with coordinates $\left(0, c_{1}\right)$ : column $c_{1}$ is determined as the column of the first pixel with non-zero value in the first row of the image.

- $\mathbf{v}_{\mathbf{2}}$ with coordinates $\left(r_{2}, M-1\right)$ : row $r_{2}$ is determined as the row of the first pixel with non-zero value in the last column of the image.

- $\mathbf{v}_{3}$ with coordinates $\left(r_{3}, 0\right)$ : row $r_{3}$ is determined as the row of the first pixel with non-zero value in the first column of the image.

- $\mathbf{v}_{4}$ with coordinates $\left(N-1, c_{4}\right)$ : column $c_{4}$ is determined as the column of the first pixel with non-zero value in the last row of the image.

Then, the lengths of each side of the image can be calculated as

$$
\begin{aligned}
& l_{1}=\left\|\mathbf{v}_{\mathbf{1}}-\mathbf{v}_{\mathbf{2}}\right\| \\
& l_{2}=\left\|\mathbf{v}_{\mathbf{2}}-\mathbf{v}_{\mathbf{3}}\right\| \\
& l_{3}=\left\|\mathbf{v}_{\mathbf{3}}-\mathbf{v}_{\mathbf{4}}\right\| \\
& l_{4}=\left\|\mathbf{v}_{\mathbf{4}}-\mathbf{v}_{\mathbf{1}}\right\|,
\end{aligned}
$$

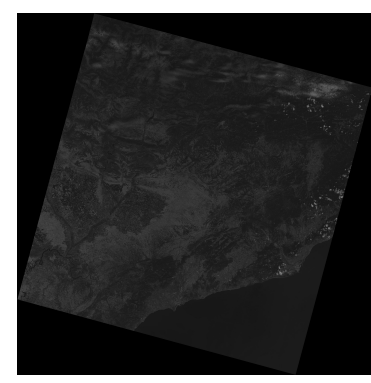

(a) Band 4

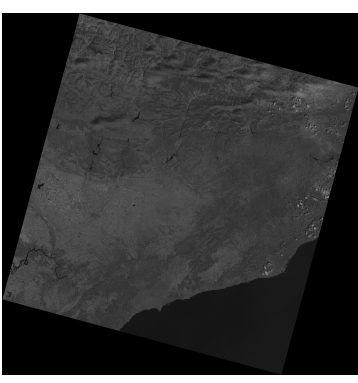

(b) Band 5

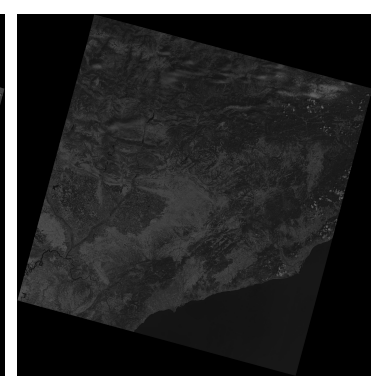

(c) Band 7

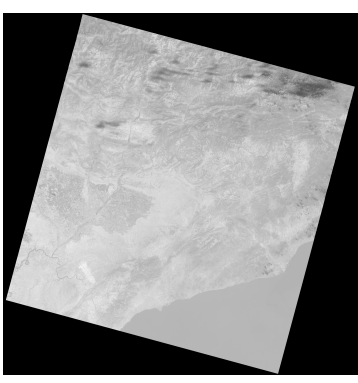

(d) Band 10

Figure 1: Landsat8 images (2019-08-14). 


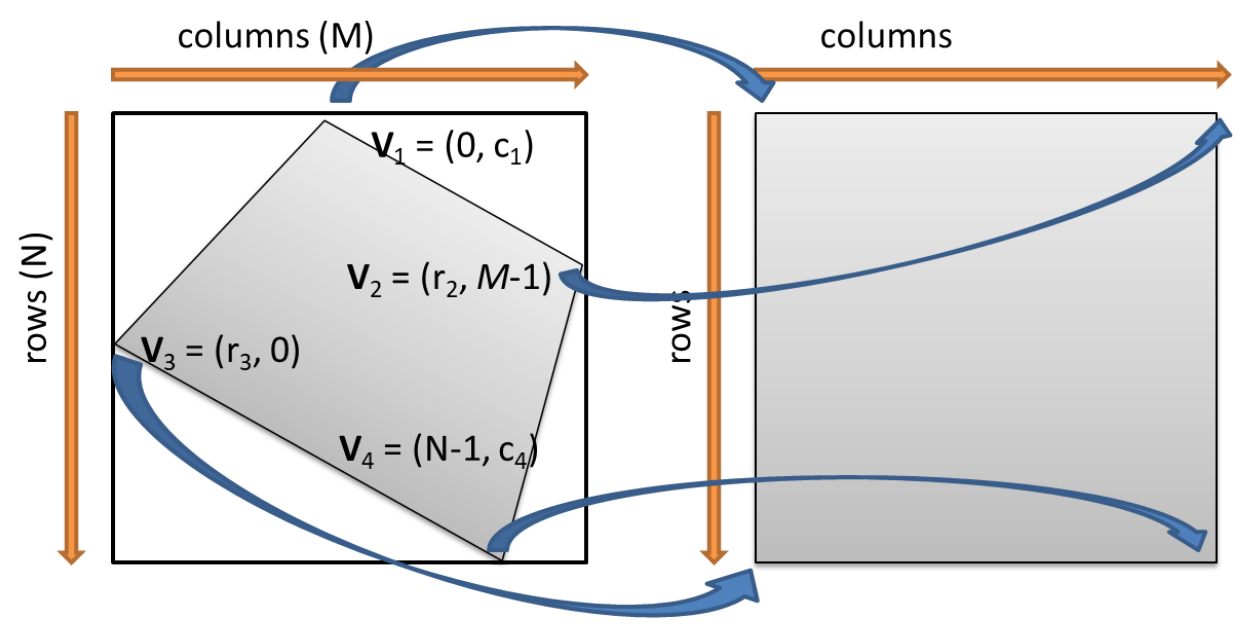

Figure 2: Information required for obtaining the homography matrix.

where $\|$.$\| stands for the norm. Note that in the present case, l_{1} \approx l_{3}$ and $l_{2} \approx l_{4}$ and thus, parallelism is preserved. Accordingly, the geometrical problem [Eq. (1)] becomes an affine transformation described by:

$$
\left(\begin{array}{c}
x^{\prime} \\
y^{\prime} \\
1
\end{array}\right)=\left(\begin{array}{ccc}
a_{11} & a_{12} & t_{x} \\
a_{21} & a_{22} & t_{y} \\
0 & 0 & 1
\end{array}\right)\left(\begin{array}{l}
x \\
y \\
1
\end{array}\right) .
$$

Then, vertices $\mathbf{v}_{\mathbf{1}}, \mathbf{v}_{\mathbf{2}}, \mathbf{v}_{\mathbf{3}}$, and $\mathbf{v}_{\mathbf{4}}$ will be transformed to a new set of vertices $\left(\mathbf{p}_{\mathbf{1}}, \mathbf{p}_{\mathbf{2}}, \mathbf{p}_{\mathbf{3}}, \mathbf{p}_{\mathbf{4}}\right)$ with coordinates:

$$
\mathbf{p}_{\mathbf{1}}=(0,0) \quad \mathbf{p}_{\mathbf{2}}=\left(\bar{l}_{x}, 0\right) \quad \mathbf{p}_{\mathbf{3}}=\left(\bar{l}_{x}, \bar{l}_{y}\right) \quad \mathbf{p}_{\mathbf{4}}=\left(0, \bar{l}_{y}\right) .
$$

The resulting images are displayed in Fig. 3. The overlaid rectangle indicates the area of interest where the fire burned. The physical length of this area is $36 \mathrm{x} 36 \mathrm{~km}^{2}$.

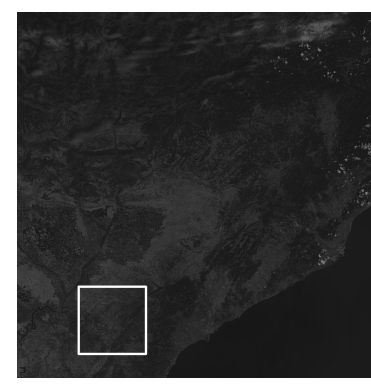

(a) Band 4

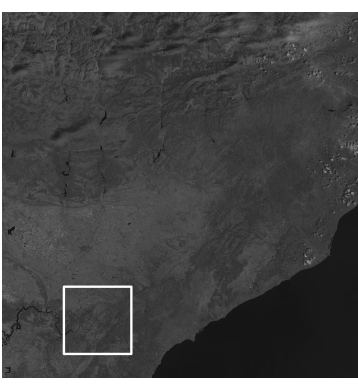

(b) Band 5

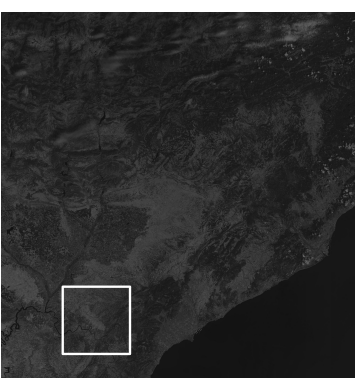

(c) Band 7

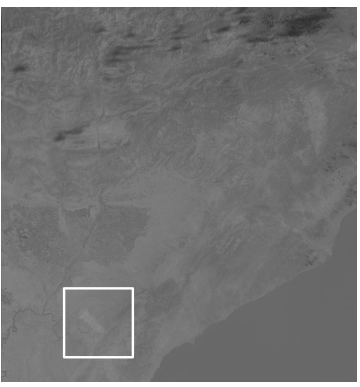

(d) Band 10

Figure 3: Geometrically-corrected images (2019-08-14). 


\section{Examination of thermal imagery}

The top of atmosphere spectral radiance $\left(L_{\lambda}\right.$, measured in $\left.\mathrm{W} \mathrm{m}^{-2} \operatorname{srad}^{-1} \mu^{-1}\right)$ can be calculated using the image recorded by the LWIR1 sensor (band 10), namely $I_{10} . L_{\lambda}$ and $I_{10}$ are related by means of

$$
L_{\lambda}=M_{L} \cdot I_{10}+A_{L}
$$

where $M_{L}=3.3420 \times 10^{-4}$ and $A_{L}=0.1$ are band-specific factors that can be found in the metadata file attached to the image set [18]. Note that $I_{10}$ is a 16 bit image with values ranging from 0 to 65535 . Now, the spectral radiance is converted to the at-sensor equivalent blackbody temperature $T_{s}$ using a model based on Planck's Law:

$$
L_{\lambda}\left(T_{s}\right)=\frac{2 h c^{2}}{\lambda^{5}} \frac{1}{e^{\frac{h c}{\lambda k_{B} T_{s}}}-1},
$$

where $k_{B}, h$ and $c$ are the Boltzmann's and Planck's constants, and the speed of light, respectively.

$T_{s}$ is determined using effective thermal constants $K_{1}=774.8853$ and $K_{2}=$ 1321.0789 that matches the behavior of the satellite sensors. This information can be found in the metadata file that accompanies the Landsat images:

$$
T_{s}=\frac{K_{2}}{\ln \left(\frac{K_{1}}{L_{\lambda}}+1\right)} \quad(\text { in } \mathrm{K}) .
$$

Note that the at-sensor temperature Ts of the burned area is close to $320 \mathrm{~K}$, as shown in Fig. 4.

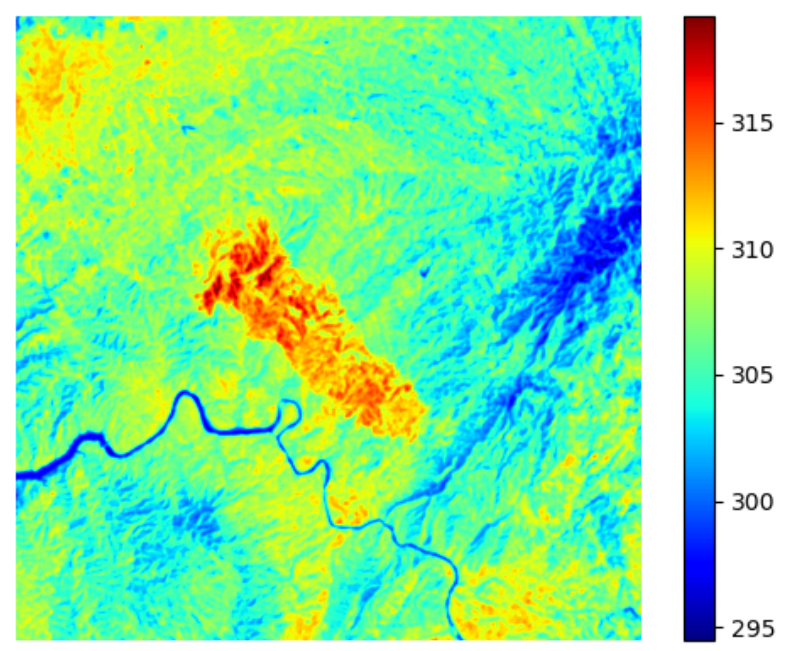

Figure 4: At-sensor temperature, $T_{s}$. 


\section{Normalized burned ratio (NBR)}

The Normalized Burn Ratio (NBR) index is introduced to assess the severity of the damage produced by fire [7]. NBR is defined as:

$$
\mathrm{NBR}=\frac{I_{5}-I_{7}}{I_{5}+I_{7}},
$$

where $I_{5}$ and $I_{7}$ are Landsat 8 images recorded on bands 5 (NIR) and 7 (SWIR) respectively. Healthy vegetation shows a very high reflectance in the near infrared NIR band and low reflectance in the SWIR. But, recently burned areas show low NIR reflectance and high SWIR reflectance. The difference between the spectral responses of healthy vegetation and burned areas reach a peak in the NIR and the SWIR regions of the spectrum.

The differential NBR (dNBR) compares data obtained before and after the wildfire. It is used to map the level of severity of the burned areas [7]: $d N B R=N B R_{\text {pre-fire }}-$ $\mathrm{NBR}_{\text {post-fire. }}$ The severity of a burned area can be assessed according to Table 2 [19]:

Table 2: Burn severity levels proposed by the USGS.

\begin{tabular}{ll}
\hline Severity level & dNBR range \\
\hline Highly enhanced regrowth & -0.500 to -0.251 \\
Slightly enhanced regrowth & -0.250 to -0.101 \\
Unburned & -0.100 to 0.099 \\
Low severity & 0.100 to 0.269 \\
Moderate-low severity & 0.270 to 0.439 \\
Moderate-high severity & 0.440 to 0.659 \\
High severity & 0.660 to 1.300 \\
\hline
\end{tabular}

Figure 5(a-d) display the calculated NBR distributions. Figures 5(a-c) show the NBR for images recorded on 2018-06-24 (pre-fire) and 2019-08-14 (post-fire), and the differential dBNR respectively. Note that the images are slightly misaligned due to the affine transform resampling used to remove those pixels with zero value., and this can lead to some errors in the dNBR calculation. The relative shift among these two image can be detected by calculating the $2 \mathrm{D}$ cross-correlation among them. The position of the cross-correlation maximum with respect to the center of the $2 \mathrm{D}$ array determines the image shift. Given two arbitrary signals $f$ and $g$, the cross-correlation product is defined as

$$
f \star g=\int_{-\infty}^{\infty} f^{*}(t) g(t+k) d t,
$$

where the symbol * stands for complex conjugate. Taking advantage of the convolution theorem, cross-correlation can be calculated in a computational efficient way as:

$$
f \star g=\mathcal{F}^{-1}\left\{\mathbf{F}^{*} \mathbf{G}\right\},
$$


where $\mathbf{F}=\mathcal{F}\{f\}$ and $\mathbf{G}=\mathcal{F}\{g\}$ are the corresponding Fourier transforms of $f$ and $g$, respectively. Figure $5(\mathrm{~d})$ displays the new version of the dNBR after removing the relative shift among the NBR images (Figs. 5(a) and 5(b)). Interestingly, Fig. 5(d) is much less noisy that Fig. 5(c)
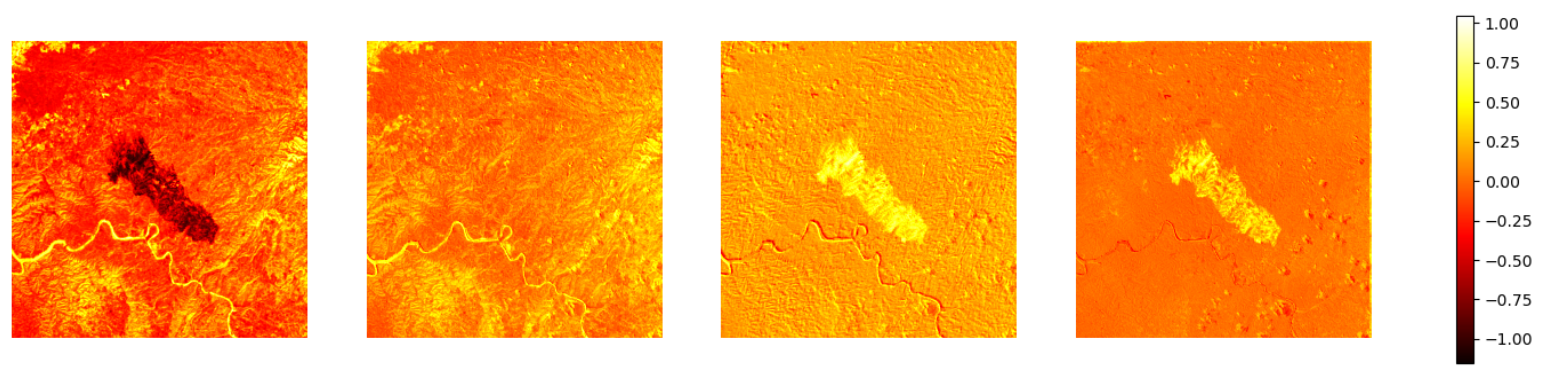

Figure 5: NBR Results: (a) NBR (2019-08-14); (b) NBR (2018-06-24); (c) dNBR (201806-24 and 2019-08-14); (d) Cross-correlation aligned dNBR (2018-06-24 and 2019-08-14). The color scale applies only to cases $(c)$ and $(d)$.

\section{Wildfire surface estimation}

To provide an estimation of the burned area, the dNBR distribution is binarized, using a threshold value equal to 0.100 (low severity damage lower bound, see Table 2). The resulting image is shown inf Fig. 6(a). It is noticeable that numerous pixels that do not belong to the burned area exceed the threshold. These pixels can be removed with the help of mathematical morphology. Let B be a binary image and $\mathrm{E}$ is the so-called structuring element. The pixels of $\mathrm{B}$ are processed one at a time. $\mathrm{E}$ is superimposed on one of the pixels of $\mathrm{B}$ in such a way that the center of array $\mathrm{E}$ determines the pixel of $\mathrm{B}$ being processed.

Erosion and dilation operators are described as follows:

(i) The resulting pixel after erosion is 1 if and only if all the pixels of $B$ that are covered by $\mathrm{E}$ are 1 ; otherwise, the pixel is set to 0 .

(ii) The resulting pixel after dilation is 1 if any of the pixels of $\mathrm{B}$ that are covered by E are 1; otherwise, the pixel becomes 0.

Opening and closing are combinations of erosion and dilation: opening is the dilation of the erosion; it is used to remove small bright objects. Closing is the erosion of the dilation, and it is useful to remove small holes. A detailed explanation on morphological techniques can be found in [16].

In the present problem, we first used a binary opening operator with a $3 \times 3$ structuring array E to discard the unconnected pixels (Fig. 6(b)). Then, the remaining isolated spots are deleted using the remove small objects function (Fig. 6(c)). This operator looks for isolated items smaller than a predetermined number of pixels (64 by 
default). Finally, we use a binary closing operator with a $5 \times 5$ structuring array E, to fill some minor gaps inside the area of interest [16].

Then, the estimation of the area burned is just the number of white pixels times the corresponding area of one pixel. We assess a burned area of $54.4 \mathrm{~km}^{2}$, slightly bigger than the official figure $\left(\gtrsim 50 \mathrm{~km}^{2}\right)$ [20].
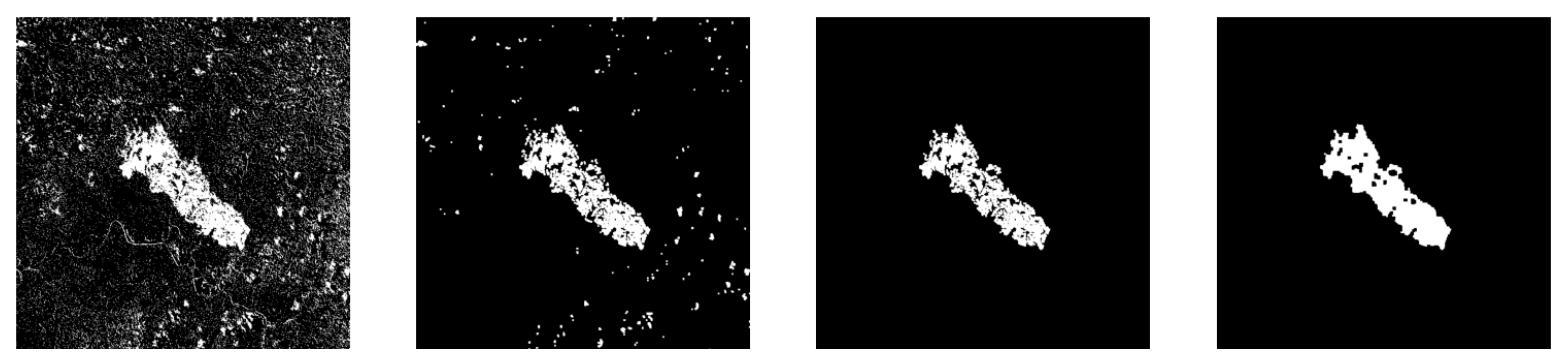

Figure 6: (a) Binary dNBR; (b) Binary dNBR after opening; (c) dNBR after opening and small objects removal; (d) dNBR after opening, small objects removal and closing.

\section{Conclusions}

In this paper we described a method to estimate the damage caused by a wildfire. Using Landsat 8 multi-spectral images recorded several days after a wildfire, we implemented a procedure to determine the temperature of the burned area. This calculation involved the combined use of infrared and visible images. Then, the differential normalized burn ratio is used to compare infrared images recorded before and after the wildfire. Using this metric and with the help of mathematical morphology techniques, we provided a reasonable estimation of the area burned.

\section{Acknowledgments}

The authors thank the US Geological Survey for the use of the Landsat 8 images. The authors are not affiliated to the USGS.

\section{Conflicts of interest}

The authors declare no conflicts of interest.

\section{References}

[1] Matthias M Boer, Víctor Resco de Dios, and Ross A Bradstock. Unprecedented burn area of australian mega forest fires. Nat. Clim. Chang., 10(3):171-172, 2020.

[2] Jennifer R Marlon, Patrick J Bartlein, Daniel G Gavin, Colin J Long, R Scott Anderson, Christy E Briles, Kendrick J Brown, Daniele Colombaroli, Douglas J Hallett, Mitchell J Power, et al. Longterm perspective on wildfires in the western usa. Proc. Natl. Acad. Sci., 109(9):E535-E543, 2012. 
[3] Marco Turco, Jost von Hardenberg, Amir AghaKouchak, Maria Carmen Llasat, Antonello Provenzale, and Ricardo M Trigo. On the key role of droughts in the dynamics of summer fires in mediterranean europe. Sci. Rep., 7(1):1-10, 2017.

[4] Vânia R Pivello. The use of fire in the cerrado and amazonian rainforests of brazil: past and present. Fire Ecol., 7(1):24-39, 2011.

[5] El Pais. Wildfire in Tarragona burns out of control after razing more than 4,000 hectares. https: //english.elpais.com/elpais/2019/06/27/inenglish/1561628514_414078.htm]. [Online; accessed 22-February-2021].

[6] U.S. Geological Survey. Landsat 8. https://www.usgs.gov/core-science-systems/nli/ landsat/landsat-8. [Online; accessed 22-February-2021].

[7] French, N H and Kasischke, E S and Hall, R J and Murphy, K A and Verbyla, D L and Hoy, E E and Allen, J L. Using Landsat data to assess fire and burn severity in the North American boreal forest region: an overview and summary of results. Int. J. Wildland Fire, 17:443, 2008.

[8] J A Richards. Remote sensing digital image analysis. Springer, 2013.

[9] L3 Harris Geospatial. Burn Indices Tutorial. https://www.13harrisgeospatial.com/docs/ BurnIndicesTutorial.html. [Online; accessed 5-July-2021].

[10] UN-Spider Knowledge Portal. Step by Step: Burn Severity mapping in Google Earth Engine. https://un-spider.org/advisory-support/recommended-practices/recommendedpractice-burn-severity/burn-severity-earth-engine. [Online; accessed 5-July-2021].

[11] Caridade, C and Encinas, A H and Martín-Vaquero, J and Queiruga-Dios, A. CAS and real life problems to learn basic concepts in linear algebra course. Comput. Appl. Eng. Educ., 23:567, 2015 .

[12] Caridade, C and Encinas, A H and Martín-Vaquero, J and Queiruga-Dios, A and Rasteiro, D. Project-based teaching in calculus courses: Estimation of the surface and perimeter of the iberian peninsula. Comput. Appl. Eng. Educ., 26:1350, 2018.

[13] Besson, U and De Ambrosis, A and Mascheretti, P. Studying the physical basis of global warming: thermal effects of the interaction between radiation and matter and greenhouse effect. Eur. J. Phys., 31:375, 2010.

[14] Xie, C and Hazzard, E. Infrared imaging for inquiry-based learning. Phys. Teach., 49:368, 2011.

[15] Shaw, J A and Nugent, P W. Physics principles in radiometric infrared imaging of clouds in the atmosphere. Eur. J. Phys., 34:S111, 2013.

[16] Gonzalez, R C and Woods, R E. Digital Image Processing. Pearson, 2018.

[17] U.S. Geological Survey. EarthExplorer. https://earthexplorer.usgs.gov/. [Online; accessed 22-February-2021].

[18] U.S. Geological Survey. Using the USGS Landsat Level-1 Data Product. https://www.usgs.gov/ land-resources/nli/landsat/using-usgs-landsat-level-1-data-product. [Online; accessed 22-February-2021].

[19] Key, C and Benson, N. Landscape Assessment (LA) Sampling and Analysis Methods. USDA Forest Service Gen. Tech. Rep. RMRS-GTR-164-CD. 200, pages LA 1-51, 2005.

[20] Goverment of Catalonia. Press release (in catalan). https://govern.cat/salapremsa/notespremsa/377331/campanya-forestal-finalitza-212-incendis-mitjana-dels-ultims-15anys-elevat-risc. [Online; accessed 22-February-2021]. 